\title{
The Reverse Reconstruction and Finite Element Analysis of the UAV Impeller
}

\author{
Chao Zhang', Changxin Kan ${ }^{2}$ \\ ${ }^{1}$ Tianjin University of Technology and Education No.1310, Dagu South Road, Hexi District, Tianjin 300222, China \\ ${ }^{2}$ Tianjin Binhai CSP Reflection Technology Co., Ltd. No. 508, 5th Floor, Building 5, NO.188 Rixin Road, Tianjin, P.R. China
}

\begin{abstract}
As a key part of the power machine, unmanned aerial vehicle (UAV) impeller is widely used in aerospace field, and it plays a very important role in the national defense industry. Impeller in the work of higher speed, centrifugal force and aerodynamic force effect on the impeller at the same time, so that the impeller is easy to failure, a serious impact on the use of performance and life of the impeller. This paper mainly studies the method of using reverse engineering technology to reconstruct the impeller of the UAV, and carries on the finite element analysis software Ansys Workbench, which satisfies the requirement of precision and intensity of impeller model.
\end{abstract}

Keywords: unmanned aerial vehicle, impeller, reverse engineering, finite element analysis, blade.

\section{Introduction}

Impeller blade is twisted, complicated curved surface, the design of impeller is more difficult, so using reverse engineering technology to get 3D model of impeller, using finite element software to perform the static strength analysis on the impeller and to test the usefulness of the reverse reconstruction of the impeller, whether can satisfy the actual demand.

\section{Reverse reconstruction of UAV impeller}

UAV impeller blade shape distortion is more complex, and the blade is thinner. Considering the characteristics of the impeller and comparing the various methods of data acquisition, choose Canada Metra SCAN 3D handheld laser scanner to get point cloud data of the impeller. It is composed of laser scanners, sensor, controller and computer, as shown in

Fig.1 (a) (b) (c), respectively.

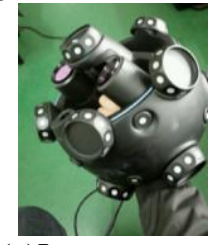

(a)Laser scanners

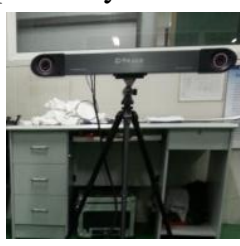

(b) sensor

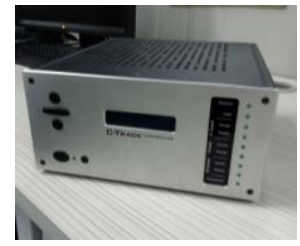

(c) controller
Figure 1: The reverse reconstruction process of UAV impeller

Pretreatment is a vital step in reverse engineering, its quality directly determines the next step the accuracy of model reconstruction. Due to some of the instrument itself, regardless of the method of contact or non-contact method is used, the measured data are inevitably produce errors occur, contain noise measured point cloud and lead to reconstruct surface produces deviation, reduce the accuracy. Non-contact measuring method has advantages of high efficiency and high precision, but its weakness is the result of the acquisition after contains a huge number of redundant data; the data display and subsequent reconstruction caused great difficulties, and will affect the contour reconstruction accuracy. So the point cloud should be pretreated. Pretreatment process mainly includes: point cloud data alignment, remove the noise point, point cloud filtering and data reduction and other processing [1].

The reconstruction of the impeller model is completed by the method of extracting feature [2], which includes the reconstruction of the wheel hub, the wheel cover, the lower end face and the blade. To reconstruct the impeller model into three coordinate measuring machine software, namely to the impeller sample as a theoretical model, of the reconstruction of the impeller model carries on the precision analysis. By the three coordinate measuring machines testing report analysis of the reconstruction of the impeller model to meet the accuracy requirements. The flow chart of the reverse reconstruction process of the unmanned aerial vehicle is shown in Figure 2.

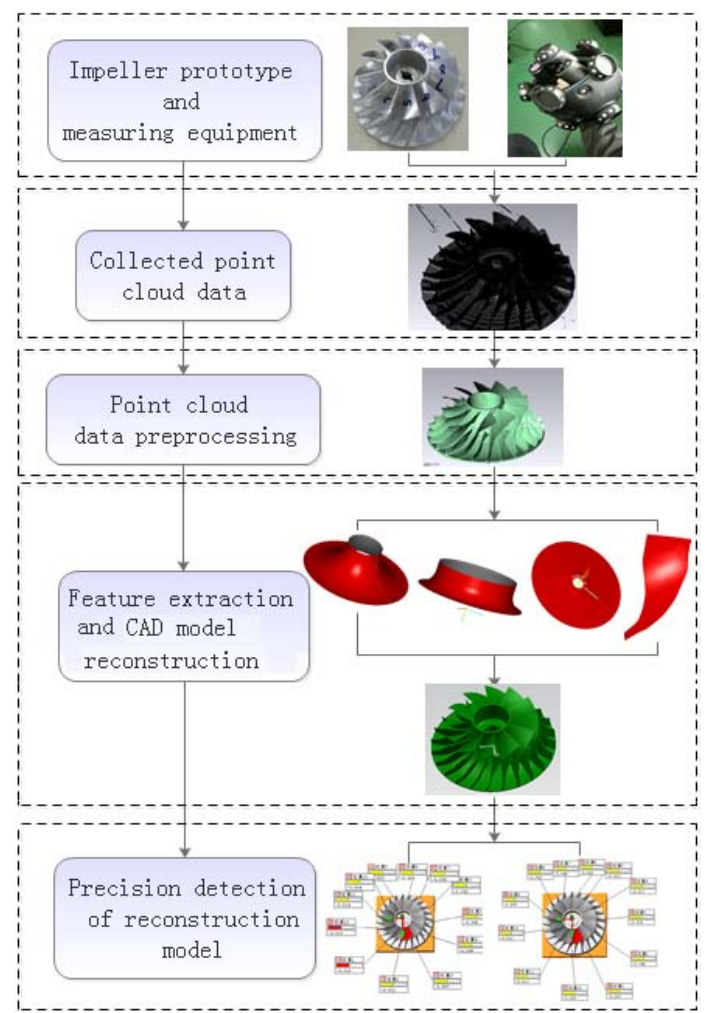

Figure 2: The reverse reconstruction process of UAV impeller 


\section{International Journal of Science and Research (IJSR) \\ ISSN (Online): 2319-7064}

Index Copernicus Value (2013): 6.14 | Impact Factor (2014): 5.611

\section{Finite Element Analysis of the UAV impeller}

\subsection{Establish analysis and finite element model}

The finite element analysis of the impeller is carried out by using ANSYS Workbench .The UAV impeller is the key component of aircraft engine. Because of its special performance, it is under the action of centrifugal force, gas pressure and thermal stress. In this paper, the effect of thermal stress on the impeller is neglected, and the influence of centrifugal force and aerodynamic force on the impeller is mainly studied.

No machine impeller a total of 24 leaves and contains 12 group, leaflets and when to perform the static strength analysis of impeller the impeller is simplified as containing only single leaf of the impeller and containing only a single leaflet of the impeller[3]. As shown in Figure 3.

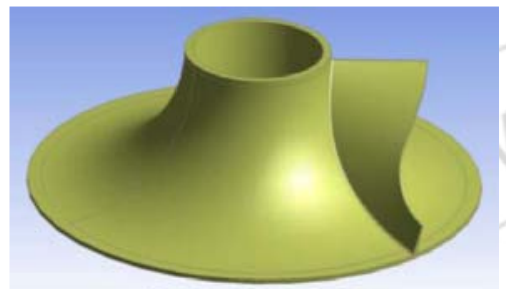

a) Analysis model of big blade impeller

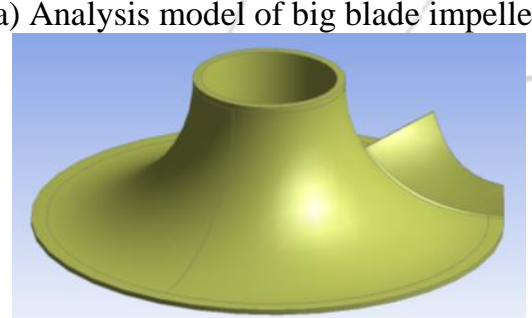

b) Analysis model of small blade impeller

Figure 3: Analysis model of the UAV impeller

The finite element model of the impeller is an indispensable part of the finite element analysis, including the setting of the material properties, the mesh generation and so on. UAV impeller material for aerospace aluminum 7075. It is a kind of high strength heat treatment alloy, has good mechanical properties, can be used well, easy to process, good wear resistance. Selection of free mesh division, with mesh metric element quality and to check the quality of the mesh is good or bad, division of grid quality abscissa from 0 to 1 , said mesh quality from good to bad, according to the local grid control to regulate the quality of the grid, dividing until the grid quality satisfy requirements[4]. After Mesh, The number of nodes and unit of big blade model is 40982 and 23502, as shown in Figure 4 (a). The number of nodes and the number of small blade model were 25424 and 13965, as shown in Figure 4 (b).

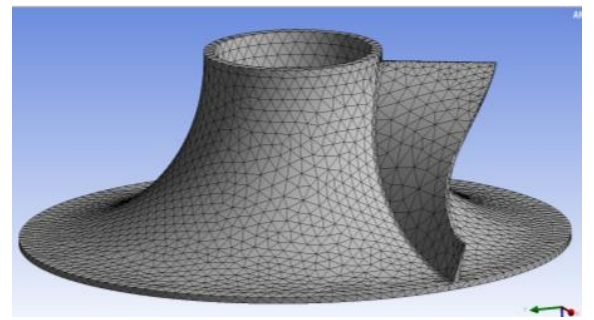

a) Finite element model of big blade impeller

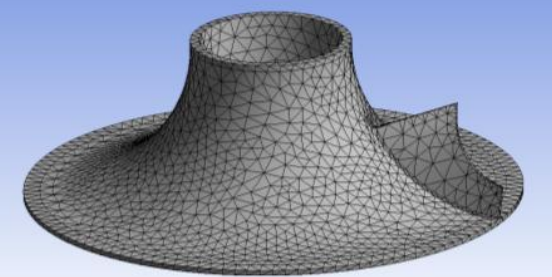

b) Finite element model of small blade impeller

Figure 4: Finite element model of impeller

\subsection{Load and boundary conditions}

Set the impeller speed is 60000r/min [5-6], can calculate the number of angular velocity of the impeller 6280rad/s. When the impeller rotating speed is high, the distribution of the corresponding force distribution is very small. The aerodynamic load value is $1 \mathrm{Mpa}$ [7-8].

In the finite element analysis of the impeller model, the constraint form of the rotating shaft hole is the same as the actual support. The boundary conditions are set to zero in all directions along the boundary. In order to avoid the rigid displacement, the node of the whole impeller model is rotated to the cylindrical coordinate system, and the hole surface of the impeller is selected, and all the displacement [9].After loading, the big and small blade impeller finite element are shown in Figure 5.

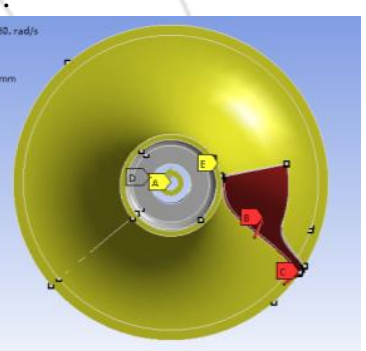

a) Finite element model of big blade after loading

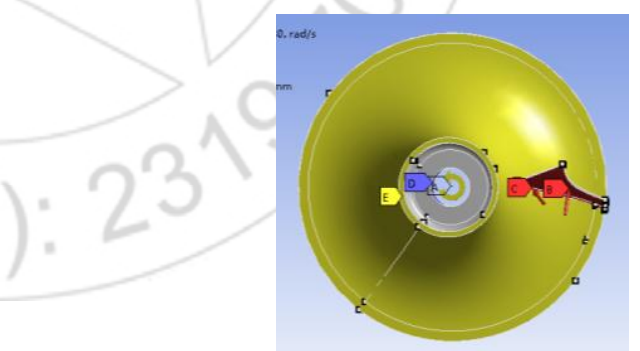

b) Finite element model of small ig blade after loading

Figure 5: Finite element model after loading

\subsection{Calculation Results and Analysis}

The UAV impeller material is 7075, the yield limit $\sigma_{s}$ is $503 \mathrm{Mpa}$, the safety factor $\mathrm{K}$ is 1.4 , and the allowable stress of the material $[\sigma]$ is $359 \mathrm{Mpa}$ calculated according to the formula[10-11].After setting the solution, start solving. The static strength analysis of impeller model, the equivalent UAV impeller stress distribution, equivalent stress and total deformation. After setting the solution, the static strength analysis of impeller model, then achieve the equivalent stress of UAV impeller. The equivalent stress and deformation are shown in Figure 6 and Figure 7. 


\section{International Journal of Science and Research (IJSR) \\ ISSN (Online): 2319-7064 \\ Index Copernicus Value (2013): 6.14 | Impact Factor (2014): 5.611}

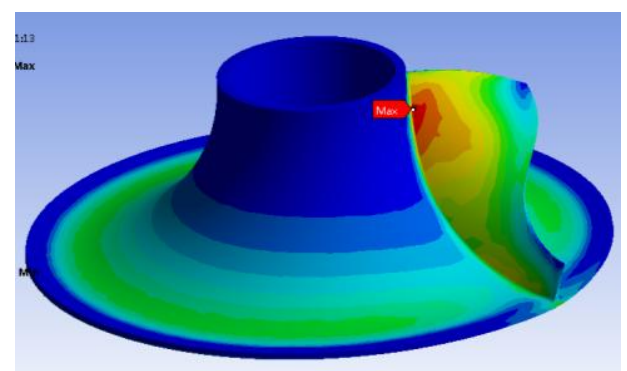

a) Equivalent stress diagram of big blade impeller model

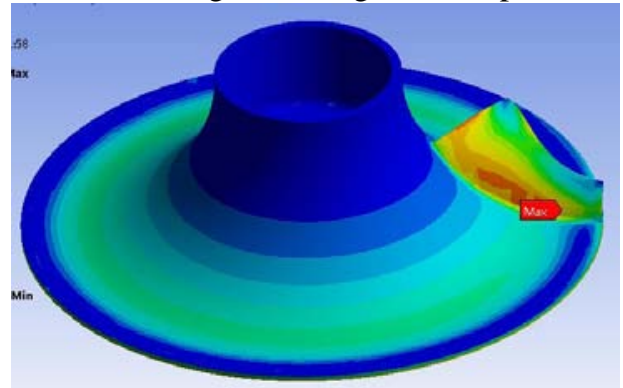

b) Equivalent stress diagram of small blade impeller model

Figure 6: Equivalent stress diagram of impeller model

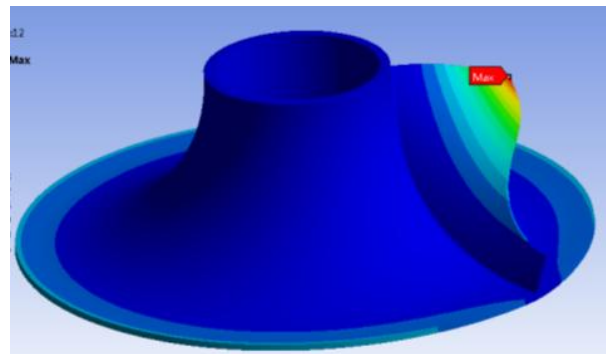

(a) Big blade impeller deformation figure

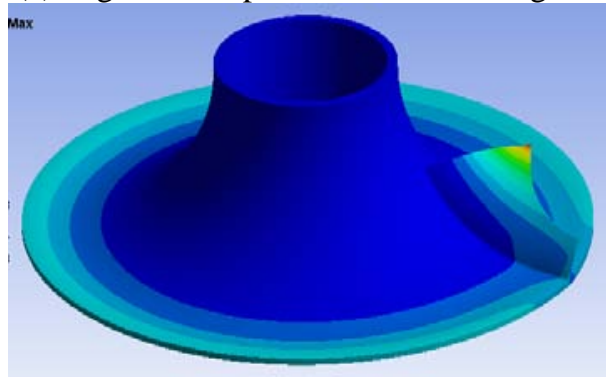

(b) Small blade impeller deformation figure

Figure 7: Deformation figure of impeller model

By the analysis, it is concluded that the analysis of all the large blade impeller, the maximum stress appears on the blade root part, small blade impeller by maximum stress occurs in the lower part of the blade root. The maximum stress values of big blade model and small blade model are 288.23Mpa and 250.69Mpa, when less than the allowable stress of material.

The maximum deformation appears at the blade tip, is also the thinnest the blade. The maximum deformation of large leaf is $0.20351 \mathrm{~mm}$, maximum deformation of the small leaf is $0.33969 \mathrm{~mm}$. So the UAVS impeller by reverse remodeling meet the strength requirements can be obtained.

\section{Conclusion}

In this paper, the method of the reverse reconstruction of the impeller is studied, and the accuracy of the model is satisfied with the three coordinate measuring machines. In the limited element software ANSYS Workbench established the finite element model of the impeller, the paper analyses the strength analysis under centrifugal loads and dynamic loads, according to the result of strength analysis, the model of UAVS impeller by reverse remodeling meet the strength requirements can be obtained.

\section{References}

[1] Qian Jinfeng. Point cloud processing in reverse engineering [D]. Hangzhou: Zhejiang University, 2005.

[2] Yan Bo, Model reconstruction and performance analysis of the impeller with Splitter Blades [D]. Nanjing: Nanjing University of Aeronautics \&amp; Astronautics, 2012.

[3] Liu Liqun. Reverse engineering and finite element analysis of turbocharger compressor impeller [D]. Shenyang: Northeastern University, 2009.

[4] Liu Jiang, etc. ANSYS 14.5 Workbench Mechanical simulation examples to explain [M]. Beijing: China Machine Press, 2014: 375-415.

[5] Zhu Ziqing, Wang Xiaolu, Chen Zeming, etc. Aerodynamic characteristics and design of unmanned aerial vehicle $[\mathrm{J}]$. Chinese Journal of Aeronautics, 2006, 27(6): 161-163.

[6] Tian Fu. Experimental research method of aerodynamic performance of the impeller [M]. Beijing: tsinghua university press, 2013: 116-135.

[7] Liu Xiaotian. ANSYS Workbench Advanced applications of structural engineering [M]. Beijing: China WaterPower Press, 2015, 50-60.

[8] Xi Guang, Wang Zhiheng, Wang Shangjin. Approximate model method and its application in aerodynamic optimization design of impeller [J]. Journal of Xi'an Jiao Tong University, 2007(2): 125-135.

[9] Li Bing, He Zhengjia, Chenxuefeng. ANSYS Workbench Design, simulation and optimization [M]. Beijing Tsinghua University press, 2008.

[10]Feng Yunxiang, Liu Jingan. Development and development of ultra high strength aluminum alloy [J]. Materials review, 2004, 18(8): 196-202.

[11] Gan Weiping, Fan Hongtao, Xu Keqin, etc. Research progress of $\mathrm{Al}-\mathrm{Zn}-\mathrm{Mg}-\mathrm{Cu}$ high strength aluminum alloy [J]. Aluminium fabrication, 2003, 150(3): 6-12.

\section{Author Profile}

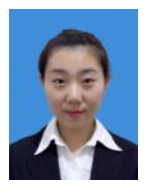

Chao Zhang is reading the M.S. degrees in Mechanical Engineering from Tianjin University of Technology and Education. 